\title{
La dimensión intercultural de las actividades de los manuales de español de los negocios del nivel B2-C1 del MCER ${ }^{1}$
}

\author{
Asliban Agdanli \\ Universitat Rovira i Virgili \\ aslihanagdanli@gmail.com
}

Resumen: El propósito del presente trabajo es dar a conocer los resultados de una investigación llevada a cabo para analizar cómo el planteamiento metodológico de las actividades de cultura de los manuales contribuyen al desarrollo de la competencia intercultural del alumno. Para ello, se han analizado siete métodos de enseñanza-aprendizaje de español de los negocios del nivel B2-C1 del MCER, publicados en España entre los años 2003 y 2010 prestando especial atención a aspectos como el procedimiento discente mediante el que se aborda el conocimiento en las actividades, las estrategias interculturales promovidas o los intercambios interculturales planteados. El análisis de los manuales me permite concluir que el planteamiento metodológico de las actividades, en general, no es adecuado para activar las habilidades y actitudes interculturales del alumno que le permitan llegar a conformar una identidad pluricultural. Por otro lado, los procesos interculturales implicados en las actividades tampoco son muy significativos para favorecer el desarrollo de la consciencia intercultural del alumno.

Palabras clave: Competencia intercultural, análisis de materiales, manuales de español de los negocios.

1 El trabajo de investigación presentado aquí parte del análisis de algunos de los datos de la tesis doctoral de la autora titulada «La comunicación intercultural en la enseñanza de español de los negocios: propuesta de un manual de español para turcos», dirigida por la doctora Sandra Iglesia Martin e inscrita en la Universitat Rovira i Virgili, Tarragona. 


\begin{abstract}
The main purpose of the present work is to show the results of a research that analyses how the methodological approach of cultural activities in foreign language textbooks contributes to the students' intercultural development skills. With this aim, seven Business Spanish course books for the levels from B2 to C1 of CEFR, published in Spain between 2003 and 2010, were analyzed. Special attention was put on aspects such as teaching procedures through which cultural knowledge is addressed within activities, promoted intercultural strategies or proposed intercultural exchange. The analysis of textbooks permits me to conclude that the methodological approach of activities, in general, is not adequate to activate the students' intercultural skills and attitudes that would allow them to define a pluricultural identity. On the other hand, the intercultural processes implied in the activities are not very significant either to promote the students' intercultural awareness development.
\end{abstract}

Key words: Intercultural competence, material analysis, business spanish textbooks. 


\section{Introducción}

En los últimos años el desarrollo de la competencia intercultural del alumno ha empezado a cobrar una considerable importancia en el mercado editorial de enseñanza de español como lengua extranjera, sobre todo en los materiales de enseñanza de español para fines específicos, más especifícamente para los negocios. Cada vez se publican más materiales en los que se incluyen secciones y/o apartados destinados a trabajar la competencia cultural y/o intercultural. Tal es el caso de la serie En equipo.es con la sección de «Diferencias culturales»; de Negocios a la vista con la sección de «Interculturalidad»; de Empresa siglo XXI con sus dos secciones de «Competencia sociocultural» $y$ «Competencia intercultural»; y de Entorno empresarial con sus cuatro unidades de cultura.

En este trabajo se presentarán algunos de los resultados obtenidos en una investigación realizada sobre el componente cultural e intercultural en los métodos de enseñanza de español de los negocios del nivel B2-C1 del MCER. El objetivo último que se pretende con esta investigación es detectar las carencias de los manuales analizados relativas al tratamiento de la cultura, para elaborar materiales didácticos complementarios que suplan las carencias encontradas, destinados a profesionales y alumnos universitarios turcos que aprenden el español para desenvolverse en contextos profesionales tanto en España como en Turquía. Como la investigación ${ }^{2}$ llevada a cabo es bastante amplia, en el presente artículo me centraré solo en una parte: el análisis de la relación entre el planteamiento metodológico de las actividades de cultura y el desarrollo de la competencia intercultural del alumno.

Los antecedentes de esta investigación se encuentran en los trabajos realizados por Areizaga (1997) que ofrece un instrumento de análisis para el componente intercultural de los métodos de enseñanza de lenguas, además de una aproximación teórica a la diferencia entre el enfoque formativo e informativo del tratamiento de la cultura; por Fernández-Conde (2005) que analiza el potencial de los manuales de español de los negocios para el desarrollo de la competencia sociocultural, con especial enfásis en la comunicación no verbal y por Riutort Canovas (2010) que ofrece un instrumento completo para analizar la competencia comunicativa intercultural de los manuales de español de los negocios y su aplicación en libros de textos de distintos niveles. El último trabajo que se mencionará aquí es el de Sercu (2000), en el que se lleva a cabo una investigación sobre la adquisición de la competencia comunicativa intercultural de los alumnos flamencos a través de los métodos de enseñanza de alemán.

2 Más datos se expondrán en próximas publicaciones. 


\subsection{El concepto de la competencia intercultural en la enseñanza-aprendizaje de lenguas extranjeras}

El desarrollo de la competencia intercultural del alumno ha sido una de las principales preocupaciones de la enseñanza de lenguas extranjeras dentro del enfoque comunicativo, sobre todo desde los años 80 y 90. La introducción de esta competencia en los currículos de enseñanza de lenguas en Europa se debe, en gran medida, al «Modelo de Competencia Comunicativa Intercultural» de Michael Byram, según el cual, la competencia comunicativa intercultural es la capacidad que tiene un individuo, como «hablante intercultural», que le permite desenvolverse con eficacia en diversas situaciones de comunicación intercultural superando los obstáculos - como malentendidos culturales, choques culturales, etc.- que suponen los encuentros interculturales (Byram, 1997).

Más tarde, el modelo de Byram se recoge con algunas modificaciones en las competencias generales del usuario descritas en el Marco Común Europeo de Referencia ${ }^{3}$ (2002), pero sin mencionar específicamente el concepto de la competencia intercultural. Cabe destacar que con la publicación de esta obra de referencia, el foco de atención en la enseñanza de lenguas se ha trasladado de la «competencia comunicativa intercultural» a la «competencia plurilingüe y pluricultural», en la cual la competencia intercultural queda como uno de los componentes de esta competencia «única y compleja» (Coste $\&$ Moore, 2009) que se describe como la capacidad que le permite al individuo vivir en una sociedad multilingüe y multicultural y abordar los desafíos comunicativos que le supone esta conviviencia (MCER, 2002).

El Plan Curricular del Instituto Cervantes ${ }^{4}$, a su vez, partiendo del concepto de la competencia plurilingüe y pluricultural y de las competencias generales del MCER (2002), define la competencia intercultural del alumno como «una capacidad de adaptarse y desenvolverse con éxito en distintas situaciones en las que se relaciona con personas de comunidades diferentes a la suya o interpreta hechos y productos culturales propios de estas comunidades» (PCIC, 2006: 598).

El tratamiento de la competencia intercultural tanto en el modelo de Byram (1997) como en los dos documentos oficiales mencionados deja muy claro que la adquisición de esta competencia supone, por un lado, el desarrollo de una serie de conocimientos culturales y una consciencia intercultural entre la cultura de origen y la cultura extranjera; y por otro lado, la puesta en juego de algunas destrezas y habilidades, y la estimulación de actitudes positivas frente a la diversidad cultural.

3 MCER, en adelante.

4 PCIC, en adelante. 
De acuerdo con esta perspectiva, no sería un error afirmar que una actividad incluida en un manual de enseñanza de lenguas extranjeras debe conllevar algunos procesos cognitivos, interculturales, conductuales y/o afectivos para poder contribuir al desarrollo de la competencia intercultural del alumno. Para tal fin, siguiendo, por un lado, el Modelo de Competencia Comunicativa Intercultural de Byram (1997) y el Modelo Metodológico de la Competencia Intercultural de Borgetti (2011) y, por otro lado, la dimensión intercultural del alumno descrita en el MCER (2002) y en el PCIC (2006) he establecido los siguientes requisitos que debe cumplir una actividad para favorecer la competencia intercultural del alumno:

- Tratar explícitamente los contenidos culturales en la actividad.

- Implicar procesos cognitivos suficientemente complejos relativos al procesamiento de los contenidos culturales.

- Ofrecer oportunidades de intercambios culturales entre la cultura extranjera y la cultura de origen del alumno sobre los aspectos culturales tratados.

- Activar, de forma estratégica, las diversas habilidades y actitudes interculturales del alumno.

Los procesos cognitivos complejos empleados en una actividad le permiten al alumno asimilar la información cultural ofrecida y trasformarla en el conocimiento cultural. Según Byram (1989: 120), la información implica la presentación arbitraria y descontextualizada de hechos con una estructura mínima y sin principios; mientras que el conocimiento se constituye de ideas, conceptos, hechos, etc., de manera estructurada, sobre el país extranjero y su gente. Durante este proceso de transición de la «información descontextualizada» al «conocimiento significativo», con la presencia de los intercambios entre la cultura de origen y la extranjera, se activan y/o estimulan diversas habilidades y actitudes interculturales que el alumno necesita poner en juego para desenvolverse en un nuevo contexto cultural. Algunas de estas habilidades y actitudes ya se encuentran disponibles en la experiencia intercultural previa del alumno; en cambio, otras aparecen como resultado del proceso de construcción del conocimiento significativo (Borghetti, 2011: 152).

Las actividades que cumplen los requisitos que se acaban de mencionar ofrecen al alumno, especialmente al que no estudia la lengua extranjera en una situación de inmersión lingüística, la oportunidad de experimentar en el aula los desafíos «extraordinarios» que afronta un extranjero continuamente en diferentes ámbitos de la vida en un país extranjero (Borghetti, 2011: 149) y esto, sin duda alguna, favorece el desarrollo de su competencia intercultural. 


\section{Análisis de los datos}

\subsection{Preguntas e hipótesis de investigación}

Partiendo de los requisitos de las actividades de cultura para favorecer el desarrollo de la competencia intercultural del alumno, se formularon las siguientes preguntas de investigación ante el análisis de la relación entre el planteamiento metodológico de estas actividades y el desarrollo de la competencia intercultural:

- ¿Las actividades de cultura de los manuales de español de los negocios del nivel B2-C1 implican procesos cognitivos suficientemente complejos para que el alumno adquiera los conocimientos culturales y promueva sus habilidades $y$ actitudes interculturales?

- ¿Los intercambios (inter)culturales que abarcan las actividades de cultura de los manuales son significativos para que el alumno desarrolle una consciencia intercultural?

- ¿Las habilidades y actitudes interculturales promovidas en las actividades son suficientemente diversas para que el alumno vaya formando una competencia intercultural?

Las hipótesis que se someterán a prueba al analizar las actividades de cultura de los manuales de español de los negocios del nivel B2-C1 son las siguientes:

Hipótesis 1: La mayor parte de las actividades de cultura de los manuales analizados no implican procesos cognitivos adecuados ni un número significativo de intercambios culturales entre la cultura meta y la cultura de origen que favorezcan, en su conjunto, el desarrollo de la competencia intercultural del alumno.

Hipótesis 2: Las habilidades y actitudes interculturales promovidas estratégicamente en la mayoría de las actividades de los manuales analizados no son suficientemente diversas para que el alumno vaya formando una competencia intercultural.

\subsection{Cuestiones metodológicas}

El estudio presentado en este artículo se basa en un análisis descriptivo y comparativo, desde lo cuantitativo, donde se emplearon tres parámetros de análisis establecidos partiendo de las preguntas e hipótesis de investigación especificadas en el apartado anterior. Con estos tres parámetros se analizaron las diferentes dinámicas de las actividades de los manuales incluidos en la investigación, y la recogida de datos se realizó a través de una base de datos diseñada con el programa Microsoft Access 2010. 


\subsection{Corpus de análisis}

La selección de los manuales incluidos en el análisis se realizó en función del área de especialidad, el nivel de lengua y la fecha de publicación del mismo. Como ya se ha adelantado en la Introducción, este análisis forma parte de un proceso de elaboración de materiales didácticos complementarios. Por esta razón, los dos primeros criterios están estrechamente relacionados con los destinatarios de estos materiales que son profesionales y alumnos universitarios turcos que aprenden el español para desenvolverse en el ámbito profesional y que poseen como mínimo el nivel B1 en este idioma.

Teniendo en cuenta las necesidades e intereses de este perfil de alumnado, la investigación se centró en los manuales de español orientados al mundo profesional, del nivel B2-C1 del MCER (2002), que reciben el nombre genérico de «manuales de español de los negocios». Respecto al criterio de la fecha de publicación del manual, se optó por analizar los manuales aparecidos después de la publicación del MCER, siendo conscientes de los cambios paradigmáticos producidos en la enseñanza-aprendizaje de lenguas extranjeras gracias a esta obra de referencia, especialmente, los relativos al desarollo de las competencias generales del alumno, que abarcan las competencias culturales e interculturales.

En el mercado editorial español se encontraron siete métodos de enseñanza de español que cumplían estos criterios establecidos para la creación del corpus de análisis. Por otro lado, el único criterio aplicado para seleccionar las actividades incluidas en el corpus fue que en la actividad se trabajara explícitamente con los contenidos culturales. De esta manera, se pretendía cumplir el primer requisito de las actividades para favorecer el desarrollo de la competencia intercultural del alumno y se dejaba el resto de los requisitos para analizar. De aquí que el corpus de análisis de este trabajo esté formado por 626 actividades y/o secuencias de actividades de los siete métodos de español de los negocios presentados en la tabla 1:

Tabla 1. Los manuales incluidos en el análisis

\begin{tabular}{|l|l|c|c|c|}
\hline \multicolumn{1}{|c|}{ Manual } & \multicolumn{1}{|c|}{ Autor(es) } & Editorial & $\begin{array}{c}\text { Fecha de } \\
\text { publicación }\end{array}$ & Nivel \\
\hline $\begin{array}{l}\text { Al di@ superior } \\
\text { Libro del alumno } \\
\text { Cuaderno de ejercicios }\end{array}$ & $\begin{array}{l}\text { Gisèle Prost, } \\
\text { Alfredo Noriega }\end{array}$ & SGEL & 2003 & B2-C1 \\
\hline $\begin{array}{l}\text { Cultura y negocios } \\
\text { (Nueva edición) } \\
\text { Libro del alumno }\end{array}$ & $\begin{array}{l}\text { Ángel Felices, Emilio Iriarte, } \\
\text { Emilia Núnez, } \\
\text { M.a Ángeles Calderón }\end{array}$ & Edinumen & 2010 & B2-C1 \\
\hline
\end{tabular}




\begin{tabular}{|l|l|l|c|c|}
\hline \multicolumn{1}{|c|}{ Manual } & \multicolumn{1}{|c|}{ Autor(es) } & Editorial & $\begin{array}{c}\text { Fecha de } \\
\text { publicación }\end{array}$ & Nivel \\
\hline $\begin{array}{l}\text { Empresa siglo XXI } \\
\text { Libro del alumno }\end{array}$ & $\begin{array}{l}\text { Emilio Iriarte, } \\
\text { Emilia Núnez Pérez }\end{array}$ & Edinumen & 2009 & B2-C1 \\
\hline $\begin{array}{l}\text { En equipo.es 3 } \\
\text { Libro del alumno } \\
\text { Cuaderno de ejercicios }\end{array}$ & $\begin{array}{l}\text { Olga Juan, Cecilia Ainciburu, } \\
\text { Ana Zaragoza, Beatriz Muñoz }\end{array}$ & Edinumen & 2007 & B2 \\
\hline $\begin{array}{l}\text { Entorno empresarial } \\
\text { Libro del alumno }\end{array}$ & $\begin{array}{l}\text { Marisa de Prada, Monserrat } \\
\text { Bovet, Pilar Marcé }\end{array}$ & Edelsa & 2008 & B2 \\
\hline $\begin{array}{l}\text { Expertos } \\
\text { Libro del alumno } \\
\text { Cuaderno de ejercicios }\end{array}$ & Marcelo Tano & Difusión & 2009 & B2 \\
\hline $\begin{array}{l}\text { Primer plano 4 } \\
\text { Libro del alumno } \\
\text { Cuaderno de ejercicios }\end{array}$ & $\begin{array}{l}\text { Germán Ruipérez García, } \\
\text { Blanca Aguirre Beltrán, } \\
\text { José Carlos García Cabrero, } \\
\text { Esperanza Román-Mendoza }\end{array}$ & Edelsa & 2003 & B2 \\
\hline
\end{tabular}

En cuanto a la cantidad de las actividades analizadas en cada manual, en la Tabla 2 se muestra la cantidad de actividades y el peso correspondiente en el corpus de análisis:

Tabla 2. Número de actividades incluidas en el análisis según los manuales

\begin{tabular}{|l|c|c|}
\hline \multicolumn{1}{|c|}{ Manuales } & Número de actividades & Su porcentaje \\
\hline Aldí@ superior, Libro del alumno & 98 & $16 \%$ \\
\hline Al dí@ superior, Cuaderno de ejercicios & 42 & $7 \%$ \\
\hline Cultura y negocios & 130 & $21 \%$ \\
\hline Empresa siglo XXI & 60 & $10 \%$ \\
\hline En equipo.es 3, Libro del alumno & 81 & $13 \%$ \\
\hline En equipo.es 3, Cuaderno de ejrecicios & 11 & $2 \%$ \\
\hline Entorno empresarial & 49 & $8 \%$ \\
\hline Expertos, Libro del alumno & 61 & $10 \%$ \\
\hline Expertos, Cuaderno de ejercicios & 31 & $5 \%$ \\
\hline Primer plano 4, Libro del alumno & 47 & $8 \%$ \\
\hline Primer plano 4, Cuaderno de ejericios & 16 & $3 \%$ \\
\hline Número total de las actividades analizadas & 626 & $100 \%$ \\
\hline
\end{tabular}

Como puede comprobarse en la tabla, el manual Cultura y negocios se representa con mayor cantidad de actividades, con 130 actividades, lo que constituye un 21\% del corpus total; está seguido por el libro del alumno del manual Al di@ superior, con 98 actividades (un 16\% del total); mientras que los cuadernos de ejercicios de los métodos En equipo.es 3 y Primer plano 4 se representan con menos actividades en el corpus — con 11 y 16 actividades respectivamente- debido al carácter puramente lingüístico de la mayor parte de sus actividades. 


\subsection{Parámetros de análisis}

De acuerdo con las preguntas e hipótesis de investigación indicadas en el apartado 2.1, se establecieron tres parámetros con los cuales se analizaron los diferentes componentes de las actividades de cultura que forman el corpus de análisis. En la Tabla 3 se presentan estos parámetros de análisis y su objetivo correspondiente:

\section{Tabla 3. Los parámetros empleados en el análisis}

\begin{tabular}{|l|l|l|}
\hline \multicolumn{1}{|c|}{ Parámetros } & \multicolumn{1}{|c|}{ Objetivo } & $\begin{array}{l}\text { Componente } \\
\text { analizado }\end{array}$ \\
\hline $\begin{array}{l}\text { 1. El procedimiento discente } \\
\text { mediante el que se aborda el } \\
\text { conocimiento cultural en la } \\
\text { actividad (Ezeiza Ramos, 2009). }\end{array}$ & $\begin{array}{l}\text { Comprobar si la actividad implica procesos } \\
\text { cognitivos suficientemente complejos para } \\
\text { asimilar el conocimiento cultural y activar } \\
\text { las estrategias interculturales del alumno. }\end{array}$ & $\begin{array}{l}\text { Componente } \\
\text { cognitivo }\end{array}$ \\
\hline $\begin{array}{l}\text { 2. Los intercambios (inter)culturales } \\
\text { implicados en la actividad (Ezeiza } \\
\text { Ramos, 2009). }\end{array}$ & $\begin{array}{l}\text { Comprobar si la actividad involucra } \\
\text { procesos interculturales que contribuyen } \\
\text { al desarrollo de la consciencia intercultural } \\
\text { del alumno. }\end{array}$ & $\begin{array}{l}\text { Componente } \\
\text { cognitivo }\end{array}$ \\
\hline $\begin{array}{l}\text { 3. Las habilidades y/o actitudes } \\
\text { promovidas en la actividad (MCER, } \\
\text { 2002; PCIC, 2006). }\end{array}$ & $\begin{array}{l}\text { Comprobar si se promueve/activa algún } \\
\text { tipo de habilidad y/o actitud intercultural } \\
\text { del alumno que necesita para desenvolverse } \\
\text { en un nuevo contexto cultural. }\end{array}$ & $\begin{array}{l}\text { Componente } \\
\text { conductual y } \\
\text { afectivo }\end{array}$ \\
\hline
\end{tabular}

1. Las variables utilizadas en este parámetro para clasificar los procedimientos discentes están extraídas de la propuesta de análisis del componente social y cultural de los materiales de enseñanza-aprendizaje de LE de Ezeiza Ramos (2009:30), y son los siguientes:

- Mediante la enseñanza explícita de aspectos relativos a la cultura de la L2.

- De forma deductiva: mediante el análisis y discusión de hechos culturales significativos o ilustrativos.

- De forma inductiva: analizando una selección de hechos culturales y tratando comprender su origen, alcance...

+ De forma contextualizada: mediante actividades de sensibilización, reconocimiento y toma de conciencia.

- A través del análisis crítico y de contraste: comparando hechos y/o usos sociales (inter)culturales nativos y no nativos, discusión de estereotipos, etc.

- Organizando tareas en torno a las actividades de la vida cotidiana y/o los usos sociales de (la L1, y) la L2.

\section{Resultados}

En este apartado se presentan los resultados obtenidos en el análisis, en dos niveles diferentes: en cada parámetro primero se exponen los resultados relativos al corpus de análisis y después, se concretan por los libros del alumno de los manuales analizados 5 .

5 Los resultados obtenidos en los cuadernos de ejercicios se han dejado fuera en este trabajo por falta de espacio; sin embargo, se presentarán en próximas publicaciones. 


\subsection{El procedimiento discente mediante el que se aborda el conocimiento cultural en la actividad}

En la Figura 1 se presenta la distribución de los procedimientos discentes empleados en las actividades del corpus de análisis para abordar los contenidos culturales:

Figura 1. La distribución de las actividades del corpus de análisis según el procedimiento discente mediante el que se aborda el conocimiento cultural

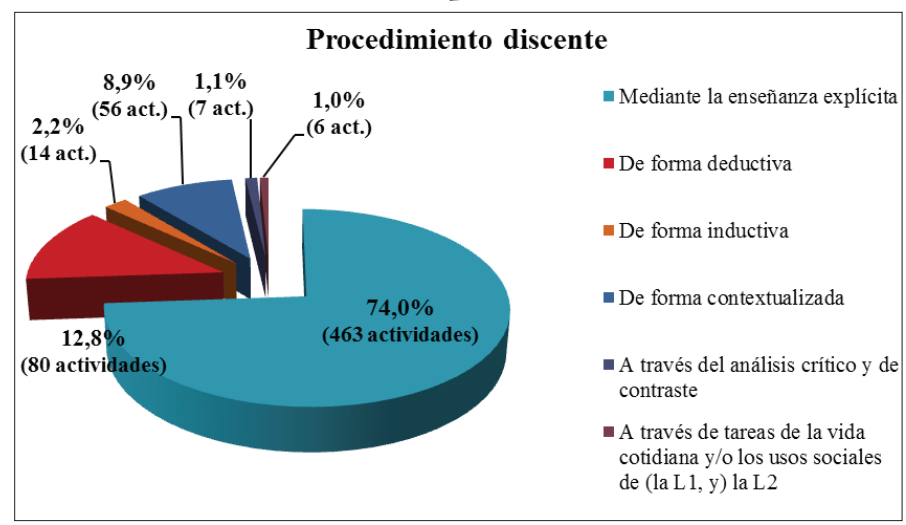

Como se puede observar en la figura anterior, «la enseñanza explícita» es el procedimiento discente preferido para abordar los contenidos culturales en las actividades que forman el corpus de análisis de este trabajo. Así, en un 74\% de las actividades del corpus de análisis se opta por este procedimiento discente. El segundo más numeroso es «la forma deductiva», empleado en un 13\% de las actividades; seguido por el procedimiento «contextualizado», es decir, a través de actividades de sensibilización, reconocimiento y toma de conciencia, utilizado en el $9 \%$ de las actividades. El resto de los procedimientos destaca por su escasa representatividad en el corpus: solo en un $2 \%$ de las actividades se abordan los contenidos culturales «de forma inductiva»; en un $1 \%$, «a través de análisis crítico y de contraste»; y en otro un 1\%, «a través de la vida cotidiana y/o usos sociales (de la L1, y) la L2».

En la Tabla 4 se presenta la distribución de los procedimientos discentes empleados en los libros del alumno analizados: 
Tabla 4. La distribución de las actividades de los libros del alumno según su procedimiento discente

\begin{tabular}{|c|c|c|c|c|c|c|c|}
\hline & 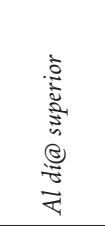 & 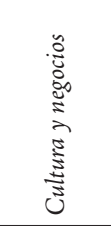 & 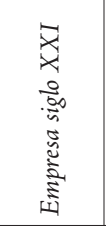 & 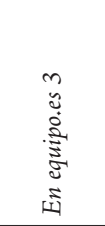 & 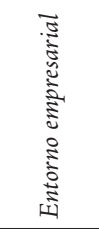 & 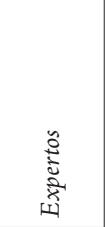 & 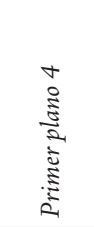 \\
\hline Mediante la enseñanza explicita & $\begin{array}{c}82 / 98 \\
(83,7 \%)\end{array}$ & $\begin{array}{l}101 / 130 \\
(77,7 \%)\end{array}$ & $\begin{array}{c}27 / 60 \\
(46,7 \%)\end{array}$ & $\begin{array}{c}57 / 81 \\
(70,4 \%)\end{array}$ & $\begin{array}{c}33 / 49 \\
(67,3 \%)\end{array}$ & $\begin{array}{c}50 / 61 \\
(82,0 \%)\end{array}$ & $\begin{array}{c}35 / 47 \\
(74,5 \%)\end{array}$ \\
\hline De forma deductiva & $\begin{array}{c}10 / 98 \\
(10,2 \%)\end{array}$ & $\begin{array}{l}8 / 130 \\
(6,2 \%)\end{array}$ & $\begin{array}{c}18 / 60 \\
(30,0 \%)\end{array}$ & $\begin{array}{c}12 / 81 \\
(14,8 \%)\end{array}$ & $\begin{array}{c}6 / 49 \\
(12,2 \%)\end{array}$ & $\begin{array}{l}5 / 61 \\
(8,2 \%)\end{array}$ & $\begin{array}{c}8 / 47 \\
(17,0 \%)\end{array}$ \\
\hline De forma inductiva & $\begin{array}{c}1 / 98 \\
(1,0 \%)\end{array}$ & $\begin{array}{l}6 / 130 \\
(4,6 \%)\end{array}$ & $\begin{array}{l}1 / 60 \\
(1,7 \%)\end{array}$ & $\begin{array}{c}1 / 81 \\
(1,2 \%)\end{array}$ & $\begin{array}{c}0 / 49 \\
(0,0 \%)\end{array}$ & $\begin{array}{c}0 / 61 \\
(0,0 \%)\end{array}$ & $\begin{array}{c}2 / 47 \\
(4,3 \%)\end{array}$ \\
\hline De forma contextualizada & $\begin{array}{l}5 / 98 \\
(5,1 \%) \\
\end{array}$ & $\begin{array}{c}12 / 130 \\
(9,2 \%)\end{array}$ & $\begin{array}{c}9 / 60 \\
(15,0 \%)\end{array}$ & $\begin{array}{c}8 / 81 \\
(9,9 \%) \\
\end{array}$ & $\begin{array}{c}10 / 49 \\
(20,4 \%) \\
\end{array}$ & $\begin{array}{c}4 / 61 \\
(6,6 \%) \\
\end{array}$ & $\begin{array}{c}2 / 47 \\
(4,3 \%)\end{array}$ \\
\hline $\begin{array}{l}\text { A través de análisis crítico } \\
y \text { de contraste }\end{array}$ & $\begin{array}{l}0 / 98 \\
(0,0 \%)\end{array}$ & $\begin{array}{l}1 / 130 \\
(0,8 \%)\end{array}$ & $\begin{array}{l}3 / 60 \\
(5,0 \%)\end{array}$ & $\begin{array}{l}1 / 81 \\
(1,2 \%)\end{array}$ & $\begin{array}{c}0 / 49 \\
(0,0 \%)\end{array}$ & $\begin{array}{l}1 / 61 \\
(1,6 \%)\end{array}$ & $\begin{array}{c}0 / 47 \\
(0,0 \%)\end{array}$ \\
\hline $\begin{array}{l}\text { A través de tareas de la vida } \\
\text { cotidiana y /o usos sociales } \\
\text { de (la L1 y la) L2 }\end{array}$ & $\begin{array}{c}0 / 98 \\
(0,0 \%)\end{array}$ & $\begin{array}{l}2 / 130 \\
(1,5 \%)\end{array}$ & $\begin{array}{l}1 / 60 \\
(1,7 \%)\end{array}$ & $\begin{array}{l}2 / 81 \\
(2,5 \%)\end{array}$ & $\begin{array}{c}0 / 49 \\
(0,0 \%)\end{array}$ & $\begin{array}{l}1 / 61 \\
(1,6 \%)\end{array}$ & $\begin{array}{c}0 / 47 \\
(0,0 \%)\end{array}$ \\
\hline
\end{tabular}

Partiendo de la tabla anterior, no sería un error afirmar que todos los métodos incluidos en el análisis tienen sus propias preferencias para abordar los contenidos culturales en sus libros del alumno. No obstante, también se observan algunas similitudes. En primer lugar, en todos los libros del alumno, «la enseñanza explícita» es el procedimiento discente preferido, a pesar de que tenga diferente importancia en cada uno; por ejemplo, en los libros del alumno de Al día superior y Expertos se emplea en más de un $80 \%$ de las actividades, mientras que en Entorno empresarial apenas llega al 70\% y en Empresa siglo XXI ni siquiera llega al $50 \%$. Asimismo, en todos los manuales, las actividades de cultura tratadas con los procedimientos discentes «de forma inductiva», «a través del análisis crítico y de contraste» o «actividades de la vida cotidiana y/o usos sociales de la L2» son escasas (en ninguno la suma de los porcentajes de estos tres procedimientos llega al 10\% del total). En Entorno empresarial, incluso, no aparecen en ninguna actividad estos procedimientos y en Al día superior, solo en una.

Las diferencias más significativas entre estos manuales se observan principalmente en el tratamiento de los contenidos culturales «de forma deductiva» $\mathrm{y}$ «de forma contextualizada» en sus libros del alumno. Por un lado, la forma deductiva es el segundo procedimiento discente preferido en los libros del alumno de $A l$ día superior, Empresa siglo XXI, En equipo.es 3, Expertos y Primer plano 4, con grandes variaciones del porcentaje. Por ejemplo, en el manual Empresa siglo XXI 
se emplea en un 30\% de las actividades; mientras que, en Expertos, solo en el 8\%. En cambio, «la forma contextualizada» es el segundo procedimiento preferido en los métodos Cultura y negocios y Entorno empresarial, con unos porcentajes de $9 \%$ y $20 \%$ respectivamente. Sin embargo, cabe añadir que Empresa siglo XXI y En equipo.es 3 disponen también buena cantidad de actividades en las que se emplea este procedimiento discente.

\subsection{Los procesos interculturales implicados en la actividad}

En la Figura 2 se exponen los resultados de los procesos (inter)culturales implicados en las actividades que forman el corpus de análisis:

Figura 2. El porcentaje de los procesos interculturales implicados en las actividades del corpus de análisis

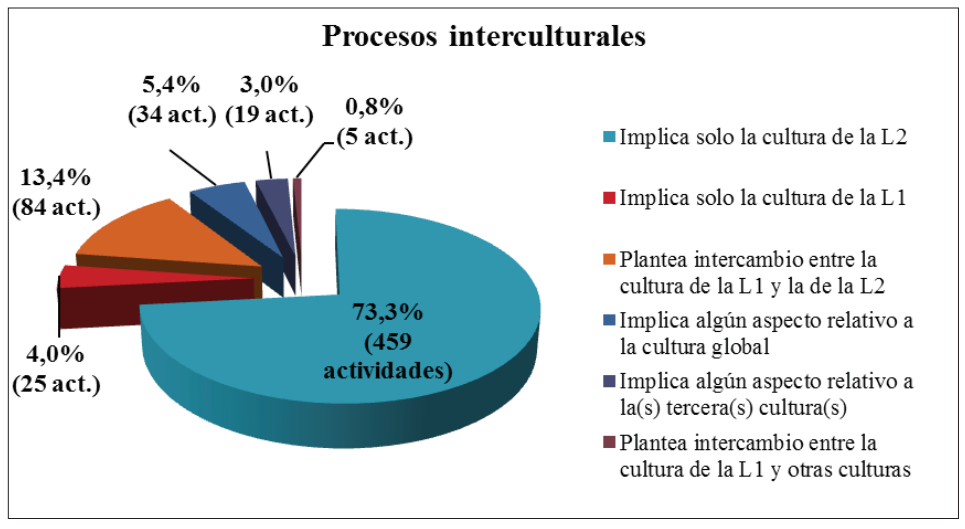

Como se puede comprobar en la figura anterior, en el 73\% de las actividades se abordan solo temas relativos a la cultura de los países hispanos; en concreto, en un $47 \%$ de las actividades, temas relativos a España; en el 17\%, relativos a los países hispanoamericanos; y en un 9\%, relativos a la vez a España (y/o a los países hispanoamericanos) y a otros países. En un 4\% de las actividades solo se tratan aspectos relativos a la cultura de origen del alumno; y en el 3\%, relativos a la cultura de otros países; por ejemplo, a los países de la UE, EEUU, China, etc., tal como se espera en un método destinado al ámbito de los negocios. En concordancia con esto, en un $5 \%$ de las actividades analizadas se abordan aspectos que pertenecen a la cultura global del mundo profesional, tal como estrategias para escribir currículos o para tener éxito en las entrevistas de trabajo, en negociaciones, etc.

Las actividades en las que se plantean intercambios entre la cultura de origen del alumno y la de los países hispanos constituyen solo un $13 \%$ de las actividades 
del corpus de análisis y, por otro lado, en un 1\% de las actividades se observan intercambios entre la cultura de origen del alumno y la cultura de otros países.

En la Tabla 5 se presentan el número total y el porcentaje correspondiente de las actividades en las que se plantean intercambios (inter)culturales entre la cultura de origen del alumno y la cultura meta, es decir, la cultura de los países hispanos en los libros del alumno analizados:

Tabla 5. La cantidad de actividades con intercambios (inter)culturales entre la cultura meta y la de origen del alumno según los manuales

\begin{tabular}{|l|c|c|}
\hline & Número de actividades & Su porcentaje \\
\hline Al dí@ superior & $16 / 98$ & $16,3 \%$ \\
\hline Cultura y negocios & $15 / 130$ & $11,5 \%$ \\
\hline Empresa siglo XXI & $5 / 60$ & $8,3 \%$ \\
\hline En equipo.es 3 & $13 / 81$ & $16,0 \%$ \\
\hline Entorno empresarial & $5 / 49$ & $10,2 \%$ \\
\hline Expertos & $11 / 61$ & $18,0 \%$ \\
\hline Primer plano 4 & $4 / 47$ & $8,5 \%$ \\
\hline
\end{tabular}

De acuerdo con lo que se acaba de decir en líneas anteriores, este tipo de actividades constituye una reducida cantidad de las actividades de cultura de los manuales objeto de análisis de este trabajo. Como se indica en la Tabla 5, los libros del alumno de los manuales $\mathrm{Al}$ día superior, En equipo.es 3 y Expertos son los que tienen relativamente más actividades de este tipo, más de un $15 \%$ de sus actividades; mientras que en Empresa siglo XXI, Entorno empresarial y Primer plano 4 apenas se encuentran 5 actividades por manual. En cuanto al manual Cultura y negocios, cuenta con 15 actividades, lo que constituye un $12 \%$ de sus actividades de cultura.

\subsection{Relación procedimiento discente-intercambios (inter) culturales en las actividades}

La Tabla 6 muestra la relación entre el procedimiento discente empleado en las actividades de corpus de análisis y los procesos interculturales implicados en estas actividades: 
Tabla 6. La relación entre el procedimiento discente adoptado en las actividades y los procesos interculturales involucrados

\begin{tabular}{|c|c|c|}
\hline Procedimiento discente empleado & $\begin{array}{c}\text { Actividades con intercambios } \\
\text { (inter)culturales }\end{array}$ & $\begin{array}{c}\text { Actividades sin intercambios } \\
\text { (inter)culturales }\end{array}$ \\
\hline Mediante la enseñanza explícita & $\begin{array}{l}59 / 463 \\
(12,7 \%) \\
\end{array}$ & $\begin{array}{r}404 / 463 \\
(87,3 \%) \\
\end{array}$ \\
\hline De forma deductiva & $\begin{array}{c}0 / 80 \\
(0,0 \%)\end{array}$ & $\begin{array}{c}80 / 80 \\
(100,0 \%)\end{array}$ \\
\hline De forma inductiva & $\begin{array}{c}2 / 14 \\
(14,3 \%)\end{array}$ & $\begin{array}{c}12 / 14 \\
(85,7 \%)\end{array}$ \\
\hline De forma contextualizada & $\begin{array}{c}22 / 56 \\
(39,3 \%)\end{array}$ & $\begin{array}{l}34 / 56 \\
(60,7 \%)\end{array}$ \\
\hline $\begin{array}{l}\text { A través de análisis crítico y de } \\
\text { contraste }\end{array}$ & $\begin{array}{c}6 / 7 \\
(83,7 \%)\end{array}$ & $\begin{array}{c}1 / 7 \\
(14,3 \%)\end{array}$ \\
\hline $\begin{array}{l}\text { A través de tareas de la vida } \\
\text { cotidiana y/o usos sociales }\end{array}$ & $\begin{array}{c}4 / 6 \\
(66,7 \%)\end{array}$ & $\begin{array}{c}2 / 6 \\
(33,3 \%)\end{array}$ \\
\hline
\end{tabular}

Como puede comprobarse en la tabla, las actividades donde los contenidos culturales se tratan «a través del análisis crítico y de contraste» $y$ «a través de actividades de la vida cotidiana y/o usos sociales de (la L1 y la) L2» son las que involucran más procesos interculturales, en un $84 \%$ y un $67 \%$ del total respectivamente; sin embargo, cabe destacar que estos dos procedimientos se representan con escasas actividades en el corpus de análisis, por esta razón, no creemos que estos sean muy representativos. Otro procedimiento que favorece bastante los procesos interculturales es «la forma contextualizada»; un 39\% de este tipo de actividades implican intercambios interculturales entre la cultura de origen del alumno y la cultura meta ( $\mathrm{y} / \mathrm{u}$ otras culturas). Por otro lado, solo en un $13 \%$ de las actividades de procedimiento explícito y en un $14 \%$ de procedimiento inductivo se plantean intercambios interculturales. En ninguna de las actividades donde los contenidos culturales se abordan «de forma deductiva» se observan intercambios interculturales, de acuerdo con su planteamiento metodológico, ya que están destinados a enseñar la cultura meta.

\subsection{Las habilidades y/o actitudes promovidas en la actividad}

En la Figura 3 se presenta la cantidad de actividades del corpus de análisis en las que se promueven las habilidades y/o actitudes interculturales del alumno: 
Figura 3. El porcentaje de actividades que promueven las habilidades y/o actitudes interculturales del alumno

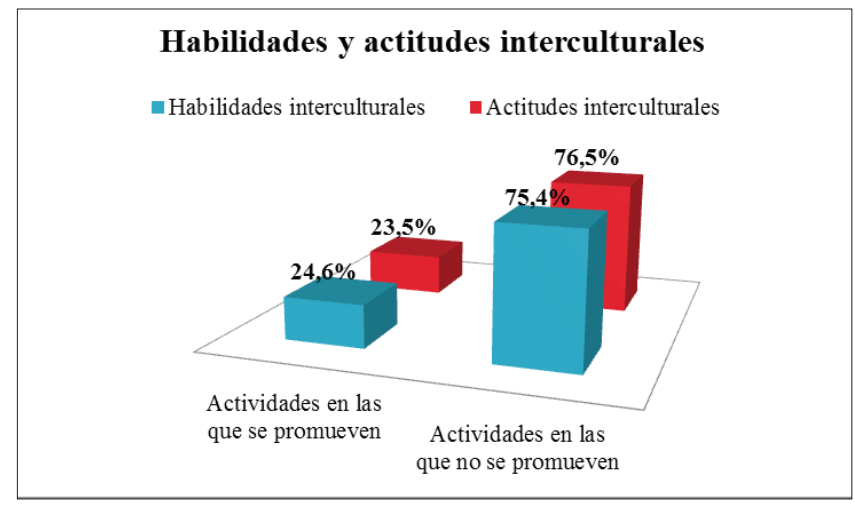

Como se puede comprobar en la figura, en un 25\% de las actividades (en 154 de las 626 actividades) del corpus de análisis de este trabajo se promueve algún tipo de habilidad intercultural del alumno; y en un 24\% (en 147 actividades en total), algún tipo de actitud intercultural.

En la Tabla 7 se clasifican las diversas habilidades y actitudes interculturales observadas en estas actividades. Es importante indicar que en una misma actividad suele implicarse más de una habilidad y/o actitud intercultural; por ejemplo, en muchos casos se observan activadas las habilidades interculturales, «Toma de conciencia y reconocimiento de la propia identidad cultural» $\mathrm{y}$ «Percepción de diferencias culturales y reconocimiento de la diversidad cultural» en la misma actividad, acompañadas con la actitud intercultural «Relativización de la propia perspectiva cultural y el propio sistema de valores». Por esa misma razón, las actividades donde se promueven las habilidades interculturales y las actividades donde se promueven las actitudes interculturales tienen unos porcentajes más o menos idénticos. 
Tabla 7. Las habilidades y actitudes interculturales promovidas en las actividades

\begin{tabular}{|l|c|c|l|}
\hline \multicolumn{1}{|c|}{ Habilidades interculturales } & \multicolumn{2}{|c|}{$\begin{array}{c}\text { Actividades en las } \\
\text { que se activan }\end{array}$} & \multicolumn{1}{|c|}{ Actitudes interculturales } \\
\hline $\begin{array}{l}\text { Toma de conciencia y } \\
\text { reconocimiento de la propia } \\
\text { identidad cultural. }\end{array}$ & $\begin{array}{c}111 / 626 \\
(17,7 \%)\end{array}$ & $\begin{array}{c}7 / 626 \\
(1,1 \%)\end{array}$ & Desarrollo de la empatía. \\
\hline $\begin{array}{l}\text { Percepción de diferencias culturales } \\
\text { y reconocimiento de la diversidad } \\
\text { cultural. }\end{array}$ & $\begin{array}{c}114 / 626 \\
(18,2 \%)\end{array}$ & $\begin{array}{c}59 / 626 \\
(9,4 \%)\end{array}$ & $\begin{array}{l}\text { Interés y apertura hacia nuevas } \\
\text { culturas y experiencias. }\end{array}$ \\
\hline $\begin{array}{l}\text { Comparación de los aspectos en los } \\
\text { que la cultura meta y la cultura de } \\
\text { origen coinciden y difieren entre sí. }\end{array}$ & $\begin{array}{c}71 / 626 \\
(11,3 \%)\end{array}$ & $\begin{array}{c}8 / 626 \\
(1,3 \%)\end{array}$ & Tolerancia a la ambigüedad. \\
\cline { 2 - 5 } & $\begin{array}{c}14 / 626 \\
(2,2 \%)\end{array}$ & Disposición favorable. \\
\hline $\begin{array}{l}\text { Cumplimiento del papel del } \\
\text { intermediario cultural. }\end{array}$ & $\begin{array}{c}7 / 626 \\
(1,1 \%)\end{array}$ & $\begin{array}{c}7 / 626 \\
(1,1 \%)\end{array}$ & $\begin{array}{l}\text { Regulación de los factores } \\
\text { afectivos en la comunicación } \\
\text { intercultural. }\end{array}$ \\
\hline $\begin{array}{l}\text { Adopción voluntaria y consciente } \\
\text { de diferentes perspectivas a la hora } \\
\text { de desenvolverse en situaciones } \\
\text { interculturales. }\end{array}$ & $\begin{array}{c}10 / 626 \\
(1,6 \%)\end{array}$ & $\begin{array}{c}114 / 626 \\
(18,2 \%)\end{array}$ & $\begin{array}{l}\text { Relativización de la propia } \\
\text { perspectiva cultural y el propio } \\
\text { sistema de valores. }\end{array}$ \\
\hline $\begin{array}{l}\text { Identificación de las causas } \\
\text { que impiden la comunicación } \\
\text { intercultural y superación de ellas. }\end{array}$ & $\begin{array}{c}11 / 626 \\
(1,8 \%)\end{array}$ & $\begin{array}{c}10 / 626 \\
(1,6 \%)\end{array}$ & $\begin{array}{l}\text { Distanciamiento de las } \\
\text { actitudes excesivamente } \\
\text { convencionales en cuanto a la } \\
\text { diferencia cultural. }\end{array}$ \\
\hline
\end{tabular}

En la tabla anterior se ve que «Toma de conciencia y reconocimiento de la propia identidad cultural»y «Percepción de diferencias culturales y reconocimiento de la diversidad cultural» son las habilidades interculturales trabajadas más frecuentemente en las actividades, puesto que se activan en más de un 18\% de actividades de este tipo. Otra habilidad intercultural muy trabajada es la «Comparación de la cultura meta y la cultura de origen» que se observa en el $11 \%$ de las actividades. En cuanto a las actitudes interculturales, «Relativización de la propia perspectiva cultural» e «Interés y apertura hacia nuevas culturas y experiencias» son las más frecuentes, ya que se encuentran activadas en un $18 \%$ y un $9 \%$ de las actividades respectivamente. El resto de las habilidades y actitudes interculturales se observan en menos de un $2 \%$ de las actividades del corpus de análisis.

La Tabla 8 muestra la cantidad de actividades donde se promueven las habilidades y actitudes interculturales del alumno incluidas en los libros del alumno analizados: 
Tabla 8. La cantidad de actividades que activan las habilidades y actitudes del alumno en los manuales analizados

\begin{tabular}{|l|c|c|c|c|}
\hline \multirow{2}{*}{} & \multicolumn{2}{|c|}{$\begin{array}{c}\text { Habilidades interculturales } \\
\text { promovidas }\end{array}$} & \multicolumn{2}{c|}{$\begin{array}{c}\text { Actitudes interculturales } \\
\text { promovidas }\end{array}$} \\
\cline { 2 - 5 } & Si & No & $S i$ & No \\
\hline \multirow{2}{*}{ Al dí@ superior } & $22 / 98$ & $76 / 98$ & $21 / 98$ & $77 / 98$ \\
& $(22,4 \%)$ & $(77,6 \%)$ & $(21,4 \%)$ & $(78,6 \%)$ \\
\hline \multirow{2}{*}{ Cultura y negocios } & $37 / 130$ & $93 / 130$ & $37 / 130$ & $93 / 130$ \\
& $(28,5 \%)$ & $(71,5 \%)$ & $(28,5 \%)$ & $(71,5 \%)$ \\
\hline \multirow{2}{*}{ Empresa siglo XXI } & $15 / 60$ & $45 / 60$ & $13 / 60$ & $47 / 60$ \\
& $(25,0 \%)$ & $(75,0 \%)$ & $(21,7 \%)$ & $(78,3 \%)$ \\
\hline \multirow{2}{*}{ En equipo.es 3 } & $21 / 81$ & $60 / 81$ & $21 / 81$ & $60 / 81$ \\
& $(25,9 \%)$ & $(74,1 \%)$ & $(25,9 \%)$ & $(74,1 \%)$ \\
\hline \multirow{2}{*}{ Entorno empresarial } & $13 / 49$ & $36 / 49$ & $13 / 49$ & $36 / 49$ \\
& $(26,5 \%)$ & $(73,5 \%)$ & $(26,5 \%)$ & $(73,5 \%)$ \\
\hline \multirow{2}{*}{ Expertos } & $16 / 61$ & $45 / 61$ & $13 / 61$ & $48 / 61$ \\
& $(26,2 \%)$ & $(73,8 \%)$ & $(21,3 \%)$ & $(78,7 \%)$ \\
\hline \multirow{2}{*}{ Primer plano 4 } & $7 / 47$ & $40 / 47$ & $7 / 47$ & $40 / 47$ \\
& $(14,9 \%)$ & $(85,1 \%)$ & $(14,9 \%)$ & $(85,1 \%)$ \\
\hline
\end{tabular}

Como se puede observar en la tabla, en este parámetro no se observan grandes diferencias entre los manuales. En todos, el porcentaje de este tipo de actividades oscila entre el 20 y 30\%, excepto en el manual Primer plano en el que apenas llega al $15 \%$. Por otro lado, el porcentaje de las actividades que trabajan con habilidades interculturales y el de las que trabajan con actitudes interculturales son idénticos en algunos de los manuales analizados, tal como Cultura y negocios, En equipo.es 3 y Entorno empresarial.

\subsection{Relación procedimiento discente-habilidades y/o actitudes interculturales}

En la Tabla 9 se muestra la cantidad de actividades en las que se activan las diferentes habilidades y/o actitudes del alumno según el procedimiento discente empleado en las mismas. Los resultados expuestos en la tabla dejan muy claro que las actividades en las que los contenidos culturales se abordan «de forma contextualizada» $y$ «a través del análisis crítico y de contraste» son las que favorecen más las habilidades y actitudes del alumno; en casi el 100\% de estas actividades se promueve algún tipo de habilidad y/o actitud intercultural del alumno. Igualmente, en una buena cantidad de las actividades del procedimiento discente «inductivo», específicamente en un $71 \%$, y en un $60 \%$ de las actividades de «la vida cotidiana y/o usos sociales de la L2» se promueven también estas habilidades y actitudes del alumno. 
Tabla 9. La cantidad de actividades en las que se activan las habilidades y/o actitudes interculturales del alumno

\begin{tabular}{|l|c|c|c|c|}
\hline \multirow{2}{*}{ Procedimiento discente empleado } & \multicolumn{2}{c|}{$\begin{array}{r}\text { Habilidades interculturales } \\
\text { promovidas }\end{array}$} & \multicolumn{2}{c|}{$\begin{array}{c}\text { Actitudes interculturales } \\
\text { promovidas }\end{array}$} \\
\cline { 2 - 5 } & Si & No & Si & No \\
\hline \multirow{2}{*}{ Mediante la enseñanza explícita } & $77 / 463$ & $386 / 463$ & $73 / 463$ & $390 / 463$ \\
& $(16,6 \%)$ & $(83,4 \%)$ & $(15,8 \%)$ & $(84,2)$ \\
\hline \multirow{2}{*}{ De forma deductiva } & $0 / 80$ & $80 / 80$ & $0 / 80$ & $80 / 80$ \\
& $(0,0 \%)$ & $(100,0 \%)$ & $(0,0 \%)$ & $(100,0 \%)$ \\
\hline \multirow{2}{*}{ De forma inductiva } & $10 / 14$ & $4 / 14$ & $10 / 14$ & $4 / 14$ \\
& $(71,4 \%)$ & $(28,6 \%)$ & $(71,4 \%)$ & $(28,6 \%)$ \\
\hline \multirow{2}{*}{ De forma contextualizada } & $56 / 56$ & $0 / 56$ & $53 / 56$ & $3 / 56$ \\
& $(100,0 \%)$ & $(0,0 \%)$ & $(94,6 \%)$ & $(5,4 \%)$ \\
\hline A través de análisis crítico y & $7 / 7$ & $0 / 7$ & $7 / 7$ & $0 / 7$ \\
de contraste & $(100,0 \%)$ & $(0,0 \%)$ & $(100,0 \%)$ & $(0,0 \%)$ \\
\hline A través de tareas de la vida cotidiana & $4 / 6$ & $2 / 6$ & $3 / 5$ & $2 / 5$ \\
y/o usos sociales de (la L1 y la) L2 & $(66,7 \%)$ & $(33,3 \%)$ & $(60,0 \%)$ & $(40,0 \%)$ \\
\hline
\end{tabular}

En cuanto al procedimiento discente «enseñanza explícita», más o menos en un $17 \%$ de las actividades donde se emplea este procedimiento se observa activado algún tipo de habilidad intercultural y/o actitud intercultural. Tal como ocurre en el caso de los procesos interculturales, en ninguna de las actividades del procedimiento deductivo se promueven las habilidades $y / o$ actitudes interculturales del alumno.

La clasificación de las habilidades interculturales activadas en las actividades según el procedimiento discente empleado se presenta en la Tabla 10, en la página siguiente. Como puede observarse en la tabla, las actividades en las que los contenidos culturales se abordan «de forma contextualizada», «de forma inductiva» $y$ «a través del análisis crítico y de contraste» no solo ofrecen más oportunidades de activar las habilidades interculturales, tal como se acaba de exponer, sino también promueven habilidades interculturales más variadas y complejas. Por ejemplo, la habilidad intercultural de «cumplir el papel intermediario entre la cultura propia y la extranjera», que aparece como uno de los objetivos generales de enseñanza de lenguas tanto en el MCER (2002) como en el PCIC (2006), solo se activa en las actividades contextualizadas y en las de análisis crítico y de contraste. 
Tabla 10. El tipo de habilidades interculturales promovidas según el procedimiento discente empleado en las actividades

\begin{tabular}{|c|c|c|c|c|c|c|}
\hline & 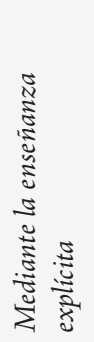 & 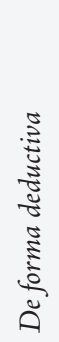 & 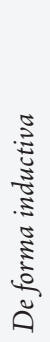 & 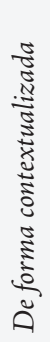 & 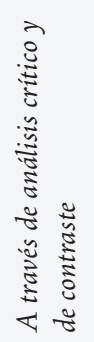 & 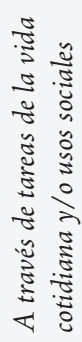 \\
\hline $\begin{array}{l}\text { Toma de conciencia y reconocimiento de } \\
\text { la propia identidad cultural. }\end{array}$ & $\checkmark$ & & $\checkmark$ & $\checkmark$ & $\checkmark$ & \\
\hline $\begin{array}{l}\text { Percepción de diferencias culturales y } \\
\text { reconocimiento de la diversidad cultural. }\end{array}$ & $\checkmark$ & & $\checkmark$ & $\checkmark$ & $\checkmark$ & $\checkmark$ \\
\hline $\begin{array}{l}\text { Comparación de los aspectos en los que } \\
\text { la cultura meta y la cultura de origen } \\
\text { coinciden y difieren entre sí. }\end{array}$ & $\checkmark$ & & $\checkmark$ & $\checkmark$ & $\checkmark$ & \\
\hline $\begin{array}{l}\text { Cumplimiento del papel del } \\
\text { intermediario cultural. }\end{array}$ & & & & $\checkmark$ & $\checkmark$ & \\
\hline $\begin{array}{l}\text { Adopción voluntaria y consciente } \\
\text { de diferentes perspectivas a la hora } \\
\text { de desenvolverse en situaciones } \\
\text { interculturales. }\end{array}$ & & & $\checkmark$ & $\checkmark$ & & $\checkmark$ \\
\hline $\begin{array}{l}\text { Identificación de las causas que } \\
\text { impiden la comunicación intercultural y } \\
\text { superación de ellas. }\end{array}$ & & & $\checkmark$ & $\checkmark$ & $\checkmark$ & \\
\hline
\end{tabular}

Tabla 11. El tipo de actitudes interculturales promovidas según el procedimiento discente empleado en las actividades

\begin{tabular}{|c|c|c|c|c|c|c|}
\hline & 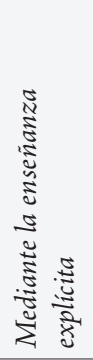 & 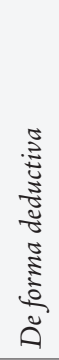 & 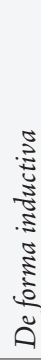 & 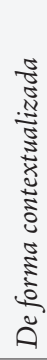 & 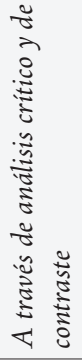 & 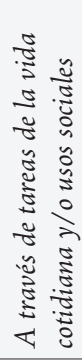 \\
\hline Desarrollo de la empatía. & & & & $\checkmark$ & $\checkmark$ & \\
\hline $\begin{array}{l}\text { Interés y apertura hacia nuevas culturas y } \\
\text { experiencias. }\end{array}$ & $\checkmark$ & & $\checkmark$ & $\checkmark$ & $\checkmark$ & \\
\hline Tolerancia a la ambigüedad. & & & & $\checkmark$ & $\checkmark$ & \\
\hline Disposición favorable. & & & $\checkmark$ & $\checkmark$ & $\checkmark$ & $\checkmark$ \\
\hline
\end{tabular}




\begin{tabular}{|l|c|c|c|c|c|c|}
\hline $\begin{array}{l}\text { Regulación de los factores afectivos en la } \\
\text { comunicación intercultural. }\end{array}$ & & & $\checkmark$ & $\checkmark$ & $\checkmark$ & \\
\hline $\begin{array}{l}\text { Relativización de la propia perspectiva } \\
\text { cultural y el propio sistema de valores. }\end{array}$ & $\checkmark$ & & $\checkmark$ & $\checkmark$ & $\checkmark$ & $\checkmark$ \\
\hline $\begin{array}{l}\text { Distanciamiento de las actitudes } \\
\text { excesivamente convencionales en cuanto a } \\
\text { la diferencia cultural. }\end{array}$ & & & $\checkmark$ & $\checkmark$ & $\checkmark$ & $\checkmark$ \\
\hline
\end{tabular}

Lo mismo sucede con las actitudes interculturales del alumno. Como puede comprobarse en la Tabla 11 las actividades donde los aspectos culturales se abordan «de forma contextualizada», «de forma inductiva» $\mathrm{y}$ «a través del análisis crítico y de contraste» favorecen el desarrollo de las actitudes interculturales más variadas. En estas actividades sobre todo se promueven las actitudes que el alumno necesita específicamente estimular a la hora de establecer contactos con extranjeros, tal como tolerancia a la ambigüedad en las relaciones interculturales, regulación de los factores afectivos en ellas, etc.

En el siguiente apartado se comentarán los resultados que se acaban de exponer y lo que suponen para la investigación llevada a cabo.

\section{Resumen y discusión de los resultados}

Los resultados obtenidos en el análisis dejan muy clara la relación entre el planteamiento metodológico de las actividades de cultura incluidas en los manuales objeto de análisis y su posible contribución al desarrollo de la competencia intercultural del alumno ${ }^{6}$. A continuación se presenta, de manera resumida, esta relación:

1. Más de un $80 \%$ de las actividades donde se tratan los contenidos culturales mediante la «enseñanza explícita» no son significativas para el desarrollo de la competencia intercultural del alumno. Estas actividades son las más tradicionales para la enseñanza de la cultura extranjera, en las cuales prima principalmente la operación cognitiva de «reconocimiento» y tampoco se plantean intercambios (inter)culturales ni se promueven habilidades $y / o$ actitudes interculturales del alumno.

El resto de las actividades construidas con este procedimiento, menos del $20 \%$, tiene potencial para contribuir a la competencia intercultural del alumno, ya que bien implican comparaciones culturales, no muy complejas, entre la cultura de origen del alumno y la cultura hispana al final de la actividad; o bien, incluyen

6 Sin embargo, es preciso indicar que el análisis llevado a cabo tiene sus propias limitaciones. Los resultados y conclusiones de este trabajo solo reflejan los casos particulares de los manuales analizados, de ninguna manera pueden generalizarse ni extenderse a los manuales no analizados. 
textos, audios, etc. que tratan temas interculturales que activan las habilidades $y$ actitudes interculturales del alumno. Sin embargo, es importante añadir que estas habilidades y actitudes promovidas no son muy variadas.

2. Las actividades en las que se tratan los contenidos culturales «de forma deductiva» en los manuales analizados, a pesar de que impliquen operaciones cognitivo-lingüísticas complejas de tipo analizar, comentar, opinar, etc. para el procesamiento de la información, no favorecen el desarrollo de la competencia intercultural del alumno, pues no involucran procesos interculturales ni activan las estrategias interculturales del alumno. Es importante precisar que en estas actividades el objeto de estudio es la cultura extranjera, no la interculturalidad y la combinación de este procedimiento con los procesos interculturales son las actividades del «análisis crítico y de contraste» que se tratarán en el ítem 5 de este apartado.

3. Las actividades con el procedimiento «inductivo» tienen más o menos la misma complejidad que las actividades del procedimiento deductivo en cuanto al procesamiento de la información. Sin embargo, a diferencia de este procedimiento, casi un $15 \%$ de sus actividades implican procesos interculturales y en un $72 \%$ se promueve una variada gama de habilidades y actitudes interculturales del alumno. Por lo tanto, puede afirmarse que este tipo de actividades, en general, contribuye al desarrollo de la competencia intercultural del alumno.

4. Lo mismo sucede con las actividades en las que los contenidos culturales se tratan «a través de tareas de la vida cotidiana y/o usos sociales de (la L1 y la) L2», este procedimiento también puede favorecer el desarrollo de la competencia intercultural del alumno, sobre todo por implicar procesos interculturales $y$ promover las estrategias interculturales del alumno en un alto porcentaje de sus actividades, casi en un $70 \%$. Sin embargo, cabe destacar que estas actividades son escasas en el corpus de análisis. Por esta razón, parece difícil cualificar en concreto su posible contribución al desarrollo de la competencia intercultural del alumno.

5. En cuanto a las actividades en las que los contenidos culturales se abordan «de forma contextualizada» $y$ «a través del análisis crítico y de contraste», además de contar con operaciones cognitivo-lingüísticas bastante complejas como cotejar, analizar, hacer hipótesis, criticar, etc.; implican un alto porcentaje de intercambios (inter)culturales $y$, al mismo tiempo, promueven diversas habilidades y actitudes interculturales del alumno. Todas estas cualidades, en su conjunto, las hacen especialmente significativas para contribuir al desarrollo de la competencia intercultural del alumno. 
De acuerdo con todo lo que se acaba de decir, los resultados obtenidos en la investigación indican que en ninguno de los libros del alumno analizados el porcentaje de las actividades significativas para el desarrollo de la competencia intercultural del alumno supera el $30 \%$ de todas las actividades de cultura incluidas. En este sentido, los manuales Empresa siglo XXI, En equipo.es 3, Entorno empresarial y Expertos son los que cuentan con unos porcentajes más altos de este tipo de actividades, que oscilan entre el 25 y el $27 \%$. Los manuales Cultura y negocios y Al día superior tienen el mismo porcentaje de actividades, un $22 \%$, apropiadas para favorecer la competencia intercultural del alumno. Por otro lado, en el manual Primer plano 4, solo un $17 \%$ de sus actividades de cultura son significativas para el desarrollo de la competencia intercultural del alumno.

De aquí que la primera hipótesis formulada para este trabajo, «la mayor parte de las actividades de cultura incluidas en los manuales analizados no implican procesos cognitivos adecuados ni un número significativo de intercambios culturales entre la cultura meta y la cultura del origen que favorezcan, en su conjunto, el desarrollo de la competencia intercultural del alumno», quede comprobada; ya que en ninguno de los manuales analizados las actividades que favorecen la competencia intercultural del alumno llega al $30 \%$ de todas las actividades de cultura, tal como se acaba de especificar en el párrafo anterior.

Este resultado coincide con las conclusiones de la investigación llevada a cabo por Riutort Cánovas (2010) sobre el desarrollo de la competencia comunicativa intercultural en los manuales de español de los negocios; según las cuales, los porcentajes de tareas destinadas al desarrollo de la conciencia intercultural son bajos o nulos en los manuales de los negocios analizados en la investigación.

Asimismo, los resultados del análisis muestran que en ninguno de los manuales analizados el porcentaje de las actividades en las que se promueven las habilidades y actitudes interculturales del alumno supera un 30\% de las actividades de cultura. Además, en la mayoría de las actividades que entran en esta franja, se suelen activar las mismas habilidades y actitudes interculturales. Las actividades con más oportunidades de activar diversas habilidades y actitudes interculturales son las que emplean los procedimientos discentes inductivo, contextualizado y el análisis crítico y de contraste. Estas actividades constituyen un 23\% de las actividades de cultura del manual Empresa siglo XXI; un 20\% de Entorno empresarial; y un $16 \%$ de Cultura y negocios. En el resto de los manuales se activan en menos del $10 \%$ de sus actividades.

Estos porcentajes indican que la segunda hipótesis sometida a prueba en la investigación, «las habilidades y actitudes interculturales promovidas estratégicamente en la mayoría de las actividades de los manuales analizados no son 
suficientemente diversas para que el alumno vaya formando una competencia intercultural», también queda comprobada.

Riutort Cánovas (2010), en su investigación mencionada anteriormente, también llega a la conclusión de que en los manuales de los negocios se observa una cobertura parcial de las habilidades interculturales del alumno. Estas coincidencias entre las dos investigaciones no sorprenden, en realidad son ciertamente esperadas, debido al hecho de que las dos investigaciones comparten una parte de su corpus de análisis que vienen de los manuales Al día superior 3, Empresa siglo $X X I$, En equipo.es 3 y Entorno empresarial.

\section{Conclusiones}

El objetivo principal de este artículo ha sido analizar la relación entre el planteamiento metodológico de las actividades de cultura de los manuales y el desarrollo de la competencia intercultural del alumno. Los resultados del análisis me permiten concluir que solo una reducida cantidad de las actividades de cultura incluidas en los manuales es significativa para el desarrollo de esta competencia, y que el resto de las actividades, carecen de procesos cognitivos e interculturales complejos que promuevan las diferentes habilidades y actitudes interculturales del alumno.

Teniendo en cuenta todo lo que se acaba de exponer, resulta difícil concluir que el alumno desarrolle una competencia intercultural que le permita relacionarse con éxito con personas de otras culturas sin tener ayuda de algún material de apoyo diseñado para este fin específico, tal como está contemplado en el MCER (2002) en relación con el desarrollo de las competencias generales del alumno.

\section{Referencias bibliográficas}

Areizaga, E. (1997). Dos décadas de enseñanza del español como lengua extranjera $a$ adultos en sus materiales. Bilbao. UPV-EHU

Borghetti, C. (2011). «How to teach it? Proposal for a Metohodological Model of Intercultural Competence». En A. White, \& T. Harden (eds.), Intercultural Competence: Concepts, Challenges, Evaluations. Bern. Peter Lang AG, International Academic Publishers: 141-159.

Byram, M. (1989). Cultural Studies in Foreign Language Education. Clevedon. Multilingual Matters. 
- (1997). Teaching and Assessing Intercultural Communicative Competence. Clevedon. Multilingual Matters.

Consejo de Europa (2002). Marco común europeo de referencia para las lenguas: aprendizaje, enseñanza, evaluación. Madrid. Anaya.

Coste, D., \& Moore, D. (2009). «Foreword». En D. Coste; D. Moore, \& G. Zarate (eds.), Plurilingual and Pluricultural Competence. Strasburg. Language Policy Division: v, vi.

Ezeiza Ramos, J. (2009). «Analizar y comprender los materiales de enseñanza en perspectiva profesional: Algunas claves para la formación del profesorado». Suplementos. MarcoELE, 9.

Fernández-Conde, M. (2005). La enseñanza de la cultura en la clase de español de los negocios. Madrid. Arco/Libros.

Instituto Cervantes (2006). Plan curricular del Instituto Cervantes: Niveles de referencia para el español (B1-B2). Madrid. Editorial Biblioteca Nueva, S.L.

Riutort Cánovas, A. (2010). La competencia comunicativa intercultural en el aula de español de los negocios: Análisis de materiales para su enseñanza. Tesis doctoral. Universidad Politécnica de Madrid, Madrid.

Sercu, L. (2000). Acquiring Intercultural Communicative Competence From Textbooks: The Case of Flemish Adolescent Pupils Learning German. Leuven (Belgium). Leuven University Press. 